\title{
Strong Surface State Effects in Nonlinear Magneto-optical Response of Ni(110)
}

\author{
K. J. Veenstra, ${ }^{1}$ A. V. Petukhov, ${ }^{1,2, *}$ E. Jurdik, ${ }^{1}$ and Th. Rasing ${ }^{1}$ \\ ${ }^{1}$ Research Institute for Materials, University of Nijmegen, Toernooiveld 1, 6525 ED Nijmegen, The Netherlands \\ ${ }^{2}$ SON Research Institute, University of Nijmegen, Toernooiveld 1, 6525 ED Nijmegen, The Netherlands
}

(Received 19 July 1999)

\begin{abstract}
Spectroscopic magnetization-induced optical second harmonic Generation (MSHG) measurements from a clean $\mathrm{Ni}(110)$ surface reveal strong resonance effects near $2.7 \mathrm{eV}$ that can be attributed to the presence of an empty surface state. The good agreement with model calculations shows the potential of MSHG to probe spin-polarized interface band structures.
\end{abstract}

PACS numbers: 78.20.Ls, 42.65.Ky, 75.70.Cn, 78.66.-w

The spin-dependent electronic structure of ferromagnetic surfaces and interfaces forms the fundamental basis for understanding magnetic phenomena such as giant magnetoresistance and interlayer exchange coupling that have attracted significant attention also because of their technological importance. Magnetic second harmonic generation (MSHG) is a new magneto-optical tool that is intrinsically sensitive to the structure and magnetism of surfaces and interfaces [1]. After its introduction in 1991 [2], MSHG has been used to study magnetic properties of, e.g., iron [3], polycrystalline nickel [4], magnetic multilayer structures (where buried layers were probed) [5-7], and to study the ultrafast magnetization dynamics in ferromagnets $[8,9]$. As the MSHG response, on a microscopic level, involves coherent electronic transitions between occupied and unoccupied electronic states in the spin-polarized band structure [10-12], MSHG is an ideal spectroscopic tool to probe the spin-polarized density of states at ferromagnetic surfaces and interfaces.

In this paper we present a phase sensitive spectroscopic MSHG [second harmonic (SH) energy 2.4-3.3 eV] investigation of a clean $\mathrm{Ni}(110)$ surface that shows that the MSHG response is sensitive to transitions from exchange split $d$ bands into empty surface states. The spectroscopic measurements were phase resolved and we show that this unique feature is essential to obtain a correct interpretation of results in the vicinity of resonances. These transitions occur around $2.7 \mathrm{eV}$ and lead to a maximum in the measured magnetic asymmetry and an exchange splitting in the magnetic tensor components. A simple model that incorporates the spin-dependent density of states gives an excellent description of the observed effects.

The second harmonic polarization $P(2 \omega)$ induced by a fundamental laser field $E(\omega)$ can be written as

$$
P_{i}(2 \omega)=\chi_{i j k}^{(2)}(-2 \omega ; \omega, \omega) E_{j}(\omega) E_{k}(\omega),
$$

with $\chi^{(2)}$ being the dipole second order nonlinear susceptibility tensor. In centrosymmetric materials this tensor is nonzero only at surfaces or interfaces where the inversion symmetry is broken [13]. The presence of a magnetization $\mathbf{M}$ breaks the time reversal symmetry and introduces odd tensor elements $\chi_{i j k}^{\text {odd }}[1]$. These tensor elements change sign upon magnetization reversal and therefore give rise to the magnetic asymmetry in the MSHG response. On a microscopic level, this asymmetry arises as in linear magnetooptics due to the splitting of the energy levels by both the exchange and the spin orbit interaction $[14,15]$. The relation between the microscopic spin-dependent band structure and the nonlinear magneto-optical susceptibility can be written as

$$
\begin{aligned}
& \chi_{i j k}^{(2)}(-2 \omega ; \omega, \omega) \\
& \quad \propto \sum_{a, b, c} \frac{\langle a|i| c\rangle\langle c|j| b\rangle\langle b|k| a\rangle}{\left(2 \hbar \omega-E_{c a}+i \hbar \Gamma_{c a}\right)\left(\hbar \omega-E_{b a}+i \hbar \Gamma_{b a}\right)},
\end{aligned}
$$

where $|a\rangle,|b\rangle$, and $|c\rangle$ are spin-dependent initial, intermediate, and final states. The measured intensity in a fixed experimental geometry with opposite magnetization directions $\pm \mathbf{M}$ can, in general, be written as a sum of effective tensor components:

$$
I^{ \pm}(2 \omega) \propto\left|\chi_{\mathrm{eff}}^{\mathrm{even}}(2 \omega) \pm \chi_{\mathrm{eff}}^{\mathrm{odd}}(2 \omega)\right|^{2},
$$

where $\chi_{\mathrm{eff}}^{\text {even }}$ and $\chi_{\mathrm{eff}}^{\text {odd }}$ are linear combinations of the even and odd tensor elements and Fresnel factors $\alpha_{i j k}$ :

$$
\chi_{\mathrm{eff}}=\sum_{i, j, k} \alpha_{i j k} \chi_{i j k} .
$$

The number of nonzero tensor elements in the summation in Eq. (4) depends upon the symmetry of the surface and the optical polarization geometry. Note that, only in the case of highly symmetric, e.g., isotropic, surfaces, it is possible to separate the odd and even contributions in the MSHG response by choosing a particular polarization combination [16]. In the case of a $\mathrm{Ni}(110)$ surface with a magnetization parallel to the easy (111) axis, the nonlinear susceptibility tensor contains 18 ( 8 even and 10 odd) independent nonzero elements [17]. The magnetic asymmetry, as measured in an MSHG measurement, can be defined as

$$
\rho=\frac{I^{+}-I^{-}}{I^{+}+I^{-}}=\frac{2\left|\chi_{\mathrm{eff}}^{\text {odd }}\right| /\left|\chi_{\text {eff }}^{\text {even }}\right|}{1+\left|\chi_{\mathrm{eff}}^{\text {odd }} / \chi_{\mathrm{eff}}^{\text {even }}\right|^{2}} \cos (\Delta \Phi) .
$$


with $\Delta \Phi$ the phase difference between the odd and even effective tensor components. Because the asymmetry $\rho$ is normalized with respect to the total SH intensity, $I^{+}+I^{-}$, it does not depend upon the intensity or shape of the fundamental light pulses, nor on the spectral properties of, e.g., filters in the optical setup. However, from Eq. (5) it follows that a measurement of only $\rho$ does not suffice to determine the spectral dependence of the effective odd and even susceptibilities separately. Therefore the relative phase between $\left|\chi_{\text {eff }}^{\text {odd }}\right|$ and $\left|\chi_{\text {eff }}^{\text {even }}\right|$ should be measured and, in addition, the intensity should be normalized to a reference.

The MSHG experiments were performed at room temperature on a disk shaped $\mathrm{Ni}(110)$ single crystal placed between the poles of an in situ electromagnet in a UHV system with a base pressure of $5 \times 10^{-11} \mathrm{mbar}$. The sample surface was cleaned by repeated cycles of $550 \mathrm{eV}$ $\mathrm{Ar}^{+}$sputtering and $e$-beam heating to $1000 \mathrm{~K}$, until no contamination could be traced by Auger-electron spectroscopy and a sharp $(1 \times 1)$ LEED (low-energy electron diffraction) pattern could be observed. For each MSHG measurement, one cleaning cycle was repeated. A tunable optical parametric amplifier pumped by a Ti-sapphire regenerative amplifier was used to produce the fundamental light pulses of $100 \mathrm{fs}$ duration in the wavelength range $840-1000 \mathrm{~nm}$ with a repetition rate of $1 \mathrm{kHz}$. Between 750 and $850 \mathrm{~nm}$, the direct output of a Ti-sapphire laser (repetition rate $82 \mathrm{MHz}$ ) was used. The second harmonic light from the Ni sample was detected with a photomultiplier tube. To normalize the measured SH intensity from the nickel, the SH intensity from a $c$-cut quartz crystal in the transmission geometry was measured with a second photomultiplier tube. Color filters (BG39) were used to filter out the fundamental light. The phase of the SH light was measured using the UHV compatible phase sensitive detection technique recently developed by us [18]. The latter is based upon interference spectroscopy where the relative phase of a sample and reference pulse, delayed with respect to each other by a time $\tau$, can be extracted from the spectrum.

The magnetic asymmetry $\rho$ measured in the polarization combination $p_{\text {in }}-p_{\text {out }}$ is plotted in Fig. 1 as a function of SH-photon energy. The open circles represent the data measured on the clean surface whereas the solid squares represent the data measured on the oxidized [0.5 L $\left(1 \mathrm{~L}=10^{-6}\right.$ Torrs) $\mathrm{O}_{2}$ ] surface. In the inset of Fig. 1, the average SH intensity $\left(I^{+}+I^{-}\right) / 2$ as measured on the clean surface is shown. $I^{+}$and $I^{-}$were measured at zero (applied) field after saturating $(0.07 \mathrm{~T})$ the $\mathrm{Ni}$ sample along the easy (111) axis. The magnetic asymmetry $\rho$ of the clean surface changes sign at 3.1 and $2.6 \mathrm{eV}$ and has a maximum at $2.7 \mathrm{eV}$. This resonant feature disappears upon oxidation, clearly proving its surface specific nature. The relation between the effective susceptibilities and the data in Fig. 1 is given by

$$
4\left|\chi_{\mathrm{eff}}\right|^{2}=I^{+}+I^{-} \pm 2 \sqrt{I^{+} I^{-}} \cos (\Delta \varphi),
$$

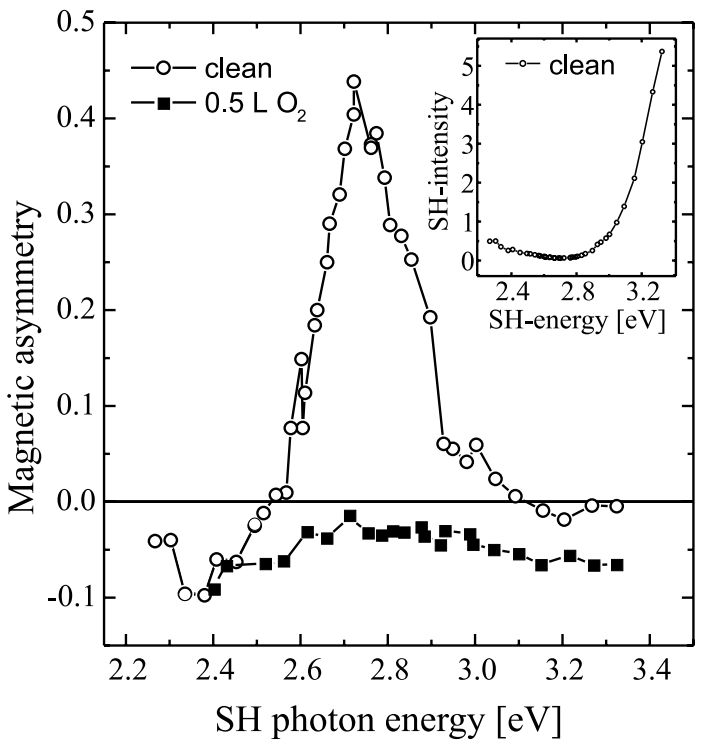

FIG. 1. Magnetic asymmetry $\rho$ as a function of second harmonic photon energy in $p_{\text {in }}-p_{\text {out }}$ polarization combination as measured on a clean and oxidized Ni(110) surface. Inset: Average second harmonic intensity as a function of second harmonic photon energy.

where $\Delta \varphi$ is the phase difference between $E(2 \omega,+\mathbf{M})$ and $E(2 \omega,-\mathbf{M})$. This phase difference has been measured as a function of frequency using the technique described in [18] and is shown in the inset of Fig. 2. The resulting effective susceptibilities $\left|\chi_{\text {eff }}^{\text {odd }}\right|$ and $\left|\chi_{\text {eff }}^{\text {even }}\right|$ are also shown in Fig. 2. Clearly, $\left|\chi_{\text {eff }}^{\text {odd }}\right|$ has two maxima at 2.82 and $2.55 \mathrm{eV}$, respectively, whereas $\left|\chi_{\mathrm{eff}}^{\mathrm{even}}\right|$ has a minimum at $2.7 \mathrm{eV}$. The error in $\chi^{\text {odd }}$ as indicated in Fig. 2 essentially results from the error in $\Delta \varphi$ which is typically $5^{\circ}$.

The resonances as observed in the nonlinear magnetooptical spectra in Figs. 1 and 2 can be explained within a simple model shown in Fig. 3. The model involves the spin splitting of the $d$ bands around the Fermi energy and an empty surface state band around $2.5 \mathrm{eV}$ above $E_{\mathrm{F}}$. The exchange splitting of the $d$ band leads to a maximum density of states for minority spin electrons at the Fermi energy and a maximum for majority spins approximately $250 \mathrm{meV}$ below $E_{\mathrm{F}}$ [19]. Several inverse photoemission studies [19-21] have reported an empty surface state band at the $\bar{Y}$ point of the fcc (110) surface Brillouin zone. As this surface state is of nearly pure $p_{z}$ character [21], the exchange splitting of this state is much smaller than the splitting of the $d$ states and can be neglected. Including only these $d$ states and surface states into the summation of Eq. (2), $\chi^{\text {odd }}$ can be written as

$$
\begin{aligned}
\chi^{\text {odd }}= & A_{0}+\frac{A_{1}}{2 \hbar \omega-E_{1}+i \hbar \Gamma_{1}} \\
& +\frac{A_{2}}{2 \hbar \omega-E_{2}+i \hbar \Gamma_{2}},
\end{aligned}
$$

where the second term describes the transitions of the minority spin electrons from filled to empty states having 


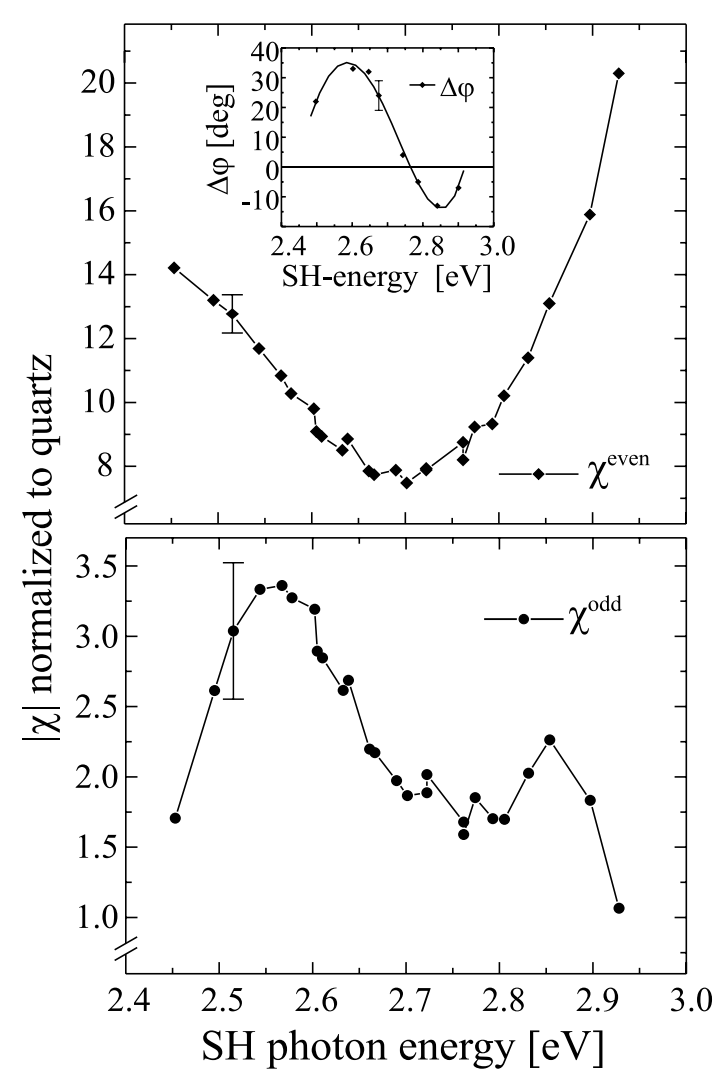

FIG. 2. Amplitude of the effective tensor elements $\chi^{\text {even }}$ and $\chi^{\text {odd }}$ as derived from the measured intensity, asymmetry, and relative phase $\Delta \varphi$. In the inset the measured frequency dependence of $\Delta \varphi$ is plotted.

energy difference $E_{1}$ and the third term includes the transitions of the majority spin electrons. $A_{1,2}$ include the matrix elements and the nonresonant energy factor from Eq. (2). $A_{0}$ is a constant background term including all nonresonant contributions. As in linear optics, this is related to an integration of all possible vertical transitions over the complete band structure. The widths of the transitions are given by $\Gamma_{1,2}$. Because of the spin dependence of the resonant contributions to the odd tensor component, the matrix elements $A_{1}$ and $A_{2}$ should have an opposite sign and are related according to $A_{1}=-|x| A_{2}$, where the factor $|x| \sim 1$ accounts for the possible difference in their absolute values.

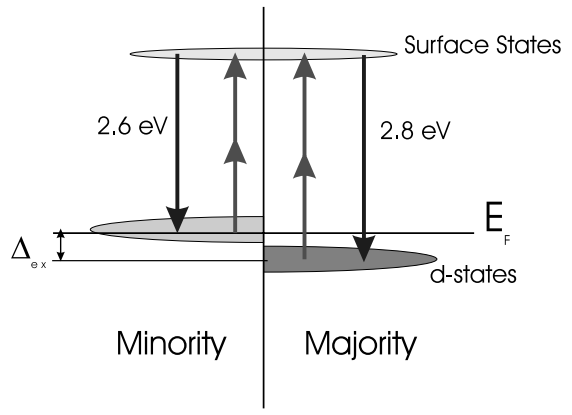

FIG. 3. Schematic picture of the exchange split density of states of nickel and empty surface states.
Note that in our model both $A_{1,2}$ and $A_{0}$ may be complex; however, for the fitting, only the relative phase of $\chi^{\text {odd }}$ is relevant and is determined by taking $A_{0}$ complex and $A_{1,2}$ real. Using the relation between $A_{1}$ and $A_{2}$, we can now fit the model to the data in Fig. 2 and obtain $\chi^{\text {odd }}$ as shown in the inset of Fig. 4 with $E_{1}=2.58 \mathrm{eV}$ and $E_{2}=2.85 \mathrm{eV}$. Once also $\chi^{\text {even }}$ is known, it is possible to check the model by calculating $\rho$ according to Eq. (5). However, $\chi^{\text {even }}$ cannot simply be described by only the transitions shown in Fig. 3. We assume that the behavior of $\chi^{\text {even }}$ is mainly determined by nonresonant contributions and partially by the resonances as indicated in Fig. 3. Because of this large nonresonant background its relative phase does not change as much as the relative phase of $\chi^{\text {odd }}$. Therefore we can fit $\chi^{\text {even }}$ simply to a real fourth rank polynomial. With this the magnetic asymmetry can now be calculated according to Eq. (5) and is shown in Fig. 4. The typical features of the asymmetry such as the two sign changes and the maximum are described very well by the model. Although the fit to $\chi^{\text {odd }}$ in Fig. 4 is not unique, to get the agreement the important physical constraint in the model is $A_{1}=-|x| A_{2}$; i.e., the two resonances must have opposite phases. If we take $A_{1}=+|x| A_{2}$ it is also possible to fit $\chi^{\text {odd }}$, however, one does not obtain a reasonable agreement for the magnetic asymmetry. This indicates that the features in $\rho$ do arise due to the difference in exchange splitting between the initial ( $d$ states) and the final (surface) states, which proves that MSHG spectroscopy, in general, can be a powerful tool to probe the spin-dependent electronic structure of surfaces and also buried interfaces.

In summary, we have measured a resonance in the nonlinear magneto-optical response from a clean $\mathrm{Ni}(110)$ surface in a UHV environment. This resonance disappears very rapidly upon oxidation of the surface (at $0.5 \mathrm{~L}$ )

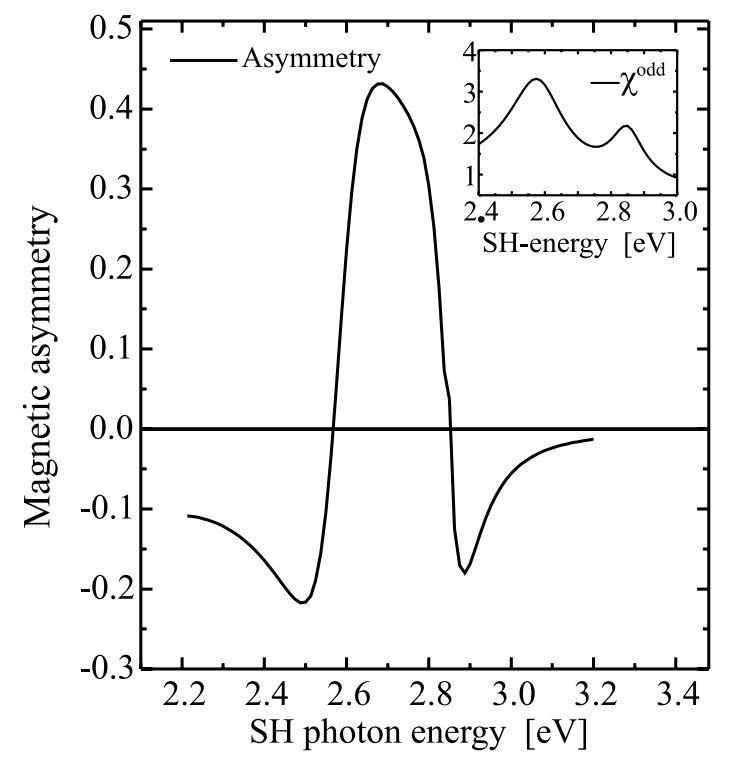

FIG. 4. Asymmetry as calculated according to the simple two Lorentzian model in Eq. (7). Inset: $\chi^{\text {odd }}$ according to the same model. 
indicating its surface state origin. By performing phase sensitive MSHG spectroscopy, we have determined the effective susceptibility spectra that can be fitted with a simple model involving the exchange splitting of $d$ states at and below the Fermi energy and an empty surface state above the Fermi energy. According to our model, the exchange splitting of the surface state should be much smaller than the splitting of the $d$ states, which confirms the $p$-like character of this surface state. Our results show that MSHG spectroscopy can indeed probe spin-dependent interface band structures. It will be very interesting to apply this technique to buried interfaces such as that between a ferromagnet and a tunnel barrier. With typical film thicknesses of a few nm, this should, in principle, be no problem.

Part of this work was supported by the Stichting Fundamenteel Onderzoek der Materie (FOM) and by the TMR network NOMOKE. Skillful assistance of A.F. van Etteger is gratefully acknowledged.

*On leave from the Institute of Crystallography, Moscow, Russia.

[1] Th. Rasing, Appl. Phys. B 68, 477 (1999).

[2] J. Reif, J. C. Zink, C.-M. Schneider, and J. Kirschner, Phys. Rev. Lett. 67, 2878 (1991).

[3] R. Vollmer, M. Straub, and J. Kirschner, Surf. Sci. 352, 937 (1996).
[4] K. Böhmer, J. Hohlfeld, and E. Matthias, Appl. Phys. A 60, 203 (1995).

[5] H. A. Wierenga et al., Phys. Rev. Lett. 74, 1462 (1995).

[6] A. Kirilyuk, Th. Rasing, R. Mégy, and P. Beauvillain, Phys. Rev. Lett. 77, 4608 (1996).

[7] A. Kirilyuk, Th. Rasing, M. Haast, and J. Lodder, Appl. Phys. Lett. 72, 2331 (1998).

[8] J. Hohlfeld, E. Matthias, R. Knorren, and K. Bennemann, Phys. Rev. Lett. 78, 4861 (1997).

[9] R. Knorren and K. H. Bennemann, Appl. Phys. B 68, 501 (1999).

[10] W. Hübner, Phys. Rev. B 42, 11553 (1990).

[11] W. Hübner, K. H. Bennemann, and K. Böhmer, Phys. Rev. B 50, 17597 (1994).

[12] U. Pustogowa, W. Hübner, and K. H. Bennemann, Phys. Rev. B 48, 8607 (1993).

[13] Y.R. Shen, The Principles of Nonlinear Optics (Wiley, New York, 1984).

[14] P. N. Argyres, Phys. Rev. 97, 334 (1955).

[15] P. Bruno, Y. Suzuki, and C. Chappert, Phys. Rev. B 53, 9214 (1996).

[16] R. Stolle et al., Phys. Rev. B 55, R4925 (1997).

[17] K. J. Veenstra et al., J. Magn. Magn. Mater. 198-199, 695 (1999).

[18] K. J. Veenstra, A. V. Petukhov, A.P. de Boer, and Th. Rasing, Phys. Rev. B 58, R16020 (1998); P. T. Wilson et al., Opt. Lett. 24, 496 (1999).

[19] M. Donath, Surf. Sci. Rep. 20, 251 (1994).

[20] A. Goldmann, V. Dose, and G. Borstel, Phys. Rev. B 32, 1971 (1985).

[21] E. Bertel, Surf. Sci. 331, 1136 (1995). 